\title{
Estimation of Input Function and Kinetic Parameters Using Simulated Annealing: Application in a Flow Model
}

\author{
Koon-Pong Wong, Member, IEEE, Steven R. Meikle, Senior Member, IEEE, Dagan Feng, Senior Member, IEEE, \\ and Michael J. Fulham
}

\begin{abstract}
Accurate determination of the input function is essential for absolute quantification of physiological parameters in positron emission tomography and single-photon emission computed tomography imaging, but it requires an invasive and tedious procedure of blood sampling that is impractical in clinical studies. We previously proposed a technique that estimates simultaneously kinetic parameters and the input function from the tissue impulse response functions and requires two blood samples. A nonlinear least squares method estimated all the parameters in the impulse response functions and the input function but failed occasionally due to high noise levels in the data, causing an ill-conditioned cost function. This paper investigates the feasibility of applying a Monte Carlo method called simulated annealing to estimate kinetic parameters in the impulse response functions and the input function. Time-activity curves of teboroxime, which is very sensitive to changes in the input function, were simulated based on published data obtained from a canine model. The equations describing the tracer kinetics in different regions were minimized simultaneously by simulated annealing and nonlinear least squares. We found that the physiological parameters obtained with simulated annealing are accurate, and the estimated input function more closely resembled the simulated curve. We conclude that simulated annealing reduces bias in the estimation of physiological parameters and determination of the input function.
\end{abstract}

Index Terms-Impulse response function, input function, kinetic parameter estimation, nonlinear least squares, simulated annealing, single-photon emission computed tomography (SPECT), teboroxime.

\section{INTRODUCTION}

A BSOLUTE quantification of dynamic positron emission tomography (PET) or single-photon emission computed tomography (SPECT) data requires an invasive procedure where

Manuscript received November 25, 2001; revised February 26, 2002. This work was supported by the National Health and Medical Research Council of Australia under Grant 980042 and by the Australian Research Council under Grant A10009011.

K.-P. Wong and S. R. Meikle are with the Department of PET and Nuclear Medicine, Royal Prince Alfred Hospital, Camperdown, NSW 2050, Australia and the School of Information Technologies, The University of Sydney, NSW 2006 Sydney, Australia (e-mail: kpong@nucmed.rpa.cs.nsw.gov.au).

D. Feng is with the Biomedical and Multimedia Information Technology Group, School of Information Technologies, The University of Sydney, NSW 2006 Sydney, Australia, and the Center for Multimedia Signal Processing, Department of Electronic and Information Engineering, The Hong Kong Polytechnic University, Hong Kong, China.

M. J. Fulham is with the Department of PET and Nuclear Medicine, Royal Prince Alfred Hospital, Missenden Road, Camperdown, NSW 2050, Australia, and the Faculty of Medicine and the School of Information Technologies, The University of Sydney, NSW 2006 Sydney, Australia.

Publisher Item Identifier S 0018-9499(02)06195-6. a series of blood samples are taken to form an input function for kinetic modeling [1]. The input function is generally obtained by sampling blood at the radial artery or from an arterialized vein in a hand. Although arterialized-venous (a-v) blood sampling has been accepted as an alternative to the gold standard of arterial blood sampling, arterial blood sampling is invasive and has the potential for irreversible tissue ischemia or arterial thrombosis. A number of methods for the estimation or elimination of the input function have been proposed [2]-[12]. The population-based input function approach [5], [7] that is used routinely at our institution calibrates a standardized input function obtained from a large population by one or two arterial or a-v blood samples for an individual by assuming that the individual input function can be closely approximated by scaling the population input function according to the area under the curve. This method has been validated in tracers with slow kinetics such as $\left[{ }^{18} \mathrm{~F}\right]$ fluorodeoxyglucose (FDG) in PET and $\left[{ }^{123} \mathrm{I}\right]$ iomazenil in SPECT [5], [7]. For tracers with fast kinetics (e.g., ${ }^{15} \mathrm{O}$-water in PET and ${ }^{99 m}$ Tc-teboroxime in SPECT), however, the population-based input function approach is unlikely to be applicable. The shape discrepancies and time delay can cause erroneous estimation of physiological parameters. Moreover, repeated measurements in a group of patients or volunteers are required for constructing a new input function template whenever a new tracer or different infusion rate is used.

Derivation of the input function from region-of-interest (ROI) placement over a vascular structure in the images has also been investigated [2], [9]. Frequent blood sampling is avoided, but the noise levels in the derived input function are high and cannot be assumed to be negligible for kinetic modeling. In addition, spillover from extravascular activity needs careful consideration, and the field of view must always include a prominent vascular structure.

The simultaneous estimation (SIME) method estimates parameters in the tissue's impulse response functions (IRFs) and the input function simultaneously and requires one or two blood samples for scaling [8], [12]. The nonlinear least squares method that has usually been regarded as the standard for kinetic modeling has been used in SIME to estimate all the parameters in the IRFs and recover the input function. However, the nonlinear least squares method fails occasionally due to ill-conditioning of the cost function caused by the high noise levels in the measurements and highly nonlinear parameter space [12]. Thus, it can be trapped into local minima, and the physiological parameters may be biased due to poor estimation of the input function. 
Although they are usually computationally demanding, minimization methods that involve only function evaluations, such as Powell's method [13], direct search [14], downhill simplex [15] and simulated annealing [16], may be able to tackle the problems associated with the nonlinear least squares method. Although simulated annealing is not an efficient optimization technique in computational terms, it is usually more reliable than other minimization methods because it is able to find the global optimum or a point very close to the global optimum in cases where other techniques fail. To our knowledge, there are few reports that apply simulated annealing to emission tomography. Most are devoted to image processing rather than to kinetic parameter estimation [17]-[19]. The aim of the study reported in this paper was to investigate the feasibility of applying simulated annealing in SIME to recover the input function and the IRFs. The combined method was evaluated with simulated cardiac dynamic SPECT ${ }^{99 \mathrm{~m}}$ Tc-teboroxime data.

\section{MATERIALS AND METHODS}

\section{A. Dynamic SPECT With ${ }^{99 \mathrm{~m}}$ Tc-Teboroxime}

Recent advances in attenuation and scatter correction methodologies in SPECT offer the possibility of quantifying the physiological functions (in vivo) by performing SPECT imaging dynamically, similar to what can be achieved with PET. One of the major applications of dynamic SPECT is to quantify myocardial perfusion, which is important for the diagnosis and clinical management of patients with coronary artery disease where a perfusion defect after an intervention may indicate incomplete reperfusion or persistent coronary occlusion. Similar to dynamic PET, compartmental modeling is used in dynamic SPECT to quantify physiological parameters of interest. It has been demonstrated that myocardial perfusion can be studied by dynamic SPECT imaging of ${ }^{99 m}$ Tc-teboroxime using multiheaded SPECT systems [20]. ${ }^{99 \mathrm{~m}}$ Tc-teboroxime is a neutral lipophilic compound with high myocardial extraction $(80-90 \%)$ in the first pass and rapid clearance [21]. Its kinetics can be modeled by a two-compartment model, as shown in Fig. 1, where $B(t)$ is the measured activity in the blood compartment at time $t, C(t)$ is the activity in the extravascular compartment at time $t$, and $k_{21}$ (in $\min ^{-1}$ ) and $k_{12}\left(\right.$ in $\mathrm{min}^{-1}$ ) are the wash-in and wash-out rate constants of teboroxime, respectively. The kinetics of teboroxime in the extravascular compartment is given by

$$
C(t)=k_{21} \int_{0}^{t} B(\tau) e^{-k_{12}(t-\tau)} d \tau
$$

with $C(0)=0$. The myocardial tissue activity, however, cannot be solely modeled by (1) because a fraction of the measured activity is contributed by the blood activity within the myocardium or nearby tissue and the partial volume effect due to the finite resolution of SPECT. Therefore, the measured myocardial tissue time-activity curve (TAC) $M(t)$ acquired between the time $t-\Delta t$ and $t$ is modeled by

$$
\hat{M}(t)=\left(1-f_{v}\right) \int_{t-\Delta t}^{t} C(\tau) d \tau+f_{v} \int_{t-\Delta t}^{t} B(\tau) d \tau
$$

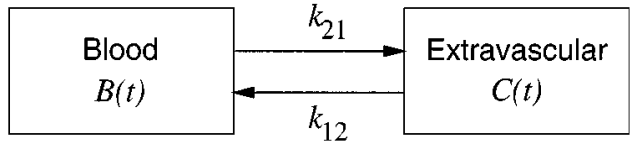

Fig. 1. Two-compartment model for ${ }^{99 m}$ Tc-teboroxime.

where $f_{v}$ represents the fraction of blood in the myocardial tissue. The parameters $k_{21}, k_{12}$, and $f_{v}$ can be estimated by nonlinear least squares fitting the measured tissue TAC to (2).

\section{B. Simultaneous Estimation}

SIME differs from other methods [3], [5], [7] that estimate or eliminate the input function based on certain properties or assumptions of the radiotracer under consideration because it uses multiple tissue TACs. These TACs are obtained by defining ROIs on the dynamic images to recover the input function embedded in the tissue TACs [8], [12]. SIME can be applied to different radiotracers or ligands, provided that the radiolabeled metabolites in blood and tissue are appropriately corrected. Since the tissue TAC is the convolution integration of the input function with the IRF of the corresponding region, the IRF parameters in multiple regions and the input function can be estimated by minimizing the residual sum of square errors between the model-predicted tissue response and the measurements in the corresponding ROIs simultaneously [8], [12]. Mathematically, the following cost function is minimized:

$$
\begin{aligned}
\Phi(\boldsymbol{p})=\sum_{i=1}^{n} \sum_{j=1}^{m} w_{t_{j}} & {\left[M_{i}\left(t_{j}\right)-\hat{M}_{i}\left(t_{j}\right)\right]^{2} } \\
& +\sum_{k=1}^{c} w_{k}\left[B\left(t_{k}\right)-\hat{B}\left(t_{k}\right)\right]^{2}
\end{aligned}
$$

subject to

$$
g(p) \geq 0
$$

where $n$ is the total number of ROIs incorporated into the model fitting procedure; $m$ is the number of data for each tissue TAC; $M_{i}\left(t_{j}\right)$ and $\hat{M}_{i}\left(t_{j}\right)$ represent the measured and model-predicted tissue activity concentrations at the $j$ th data point in the $i$ th ROI; $w_{t_{j}}$ is the weight associated with the $j$ th measurement in the tissue TAC and is chosen to be the inverse of the variance of the corresponding measurement; $\boldsymbol{p}$ denotes the vector of parameters to be estimated, including the wash-in and wash-out parameters in multiple tissue TACs and the estimated input function $\hat{B}(t)$; $\boldsymbol{g}(\boldsymbol{p})$ is a set of physiological (inequality) constraints imposed on $\boldsymbol{p} ; c$ is the number of arterial blood samples taken late in the course of the study for calibration; $B\left(t_{k}\right)$ is the activity concentration in blood measured at time $t_{k}(k=1,2, \ldots, c)$; and $w_{k}$ is chosen to be 100 (or any other reasonably large value) so that the blood samples are given more weight to discourage any discrepancy between the measurements and their predictions. Note that $B(t)$ in (1) and (2) is replaced by $\hat{B}(t)$ when minimizing (3) because the input function is estimated rather than measured.

\section{Nonlinear Least Squares}

The principle of the nonlinear least squares method is to iteratively minimize a cost function based on a least squares cri- 
terion. Rapid convergence can be achieved if the shape of the isocontours of the cost function is approximately a concentric circle and can be well approximated by a quadratic around the minimum. In this study, the Marquardt algorithm [22] was used, as it usually performs well in locating the minimum and is reasonably insensitive to an initial estimate. However, there is no guarantee that the located minimum corresponds to the global minimum unless it is unique. Furthermore, in optimization problems with a large number of parameters or with noisy data, the cost function is usually ill-conditioned because it has several minima. In this case, the algorithm is likely to get stuck in the local minimum nearest to the initial estimate, or in the worst case, the algorithm does not converge if the cost function is nondifferentiable or discontinuous in its domain.

\section{Simulated Annealing}

Simulated annealing was developed by Kirkpatrick et al. [16]. It is a generalization of a Monte Carlo method introduced by Metropolis et al. based on the theory of statistical mechanics [23]. In condensed matter physics, annealing is a physical process of heating up a solid material by increasing the temperature to a maximum at which all molecules arrange themselves randomly in the liquid phase, followed by a slow cooling process that results in the formation of a perfect crystal. Application of simulated annealing to optimization problems is based on the analogy between the state of each molecule and the state of each parameter that affects the energy function (analogous to the cost function in the optimization problem) to be minimized. The parameter values are randomly perturbed, and the probability of accepting the perturbed cost function is determined by the Metropolis criterion [16], [23]

$$
P(\Delta \Phi)= \begin{cases}1, & \text { if } \Delta \Phi \leq 0 \\ e^{-\frac{\Delta \Phi}{K_{B} T}}, & \text { otherwise }\end{cases}
$$

where $\Delta \Phi$ is the change in the cost function due to the perturbation, $K_{B}$ is the Boltzmann constant, and $T$ is the current system temperature, which is a control parameter [16]. As the iterations progress, the temperature parameter $T$ is reduced. Providing that the starting maximum temperature is sufficiently high and the temperature $T$ is lowered slowly, the algorithm is guaranteed to reach the global minimum or a point close to the global minimum of the cost function [16], [23].

Implementation of the simulated annealing algorithm is relatively straightforward. We followed the implementation described by Corana et al. [24] but introduced the interior-point method [25] to minimize (3), which actually defined an inequality-constrained minimization problem. The interior-point method [25] enforces the physiological (inequality) constraints imposed on the kinetic parameters by creating a sequence of modified functions whose unconstrained minimum should converge to the constrained minimum in the limit [25]. The convergence of this method is considered beyond the scope of this paper.

Fig. 2 shows a simplified flow chart of the modified simulated annealing algorithm. Starting with a given set of initial parameters $\boldsymbol{p}$ and a high initial temperature $T\left(=10^{9}\right)$, the cost function is then calculated and recorded. The current parameters are

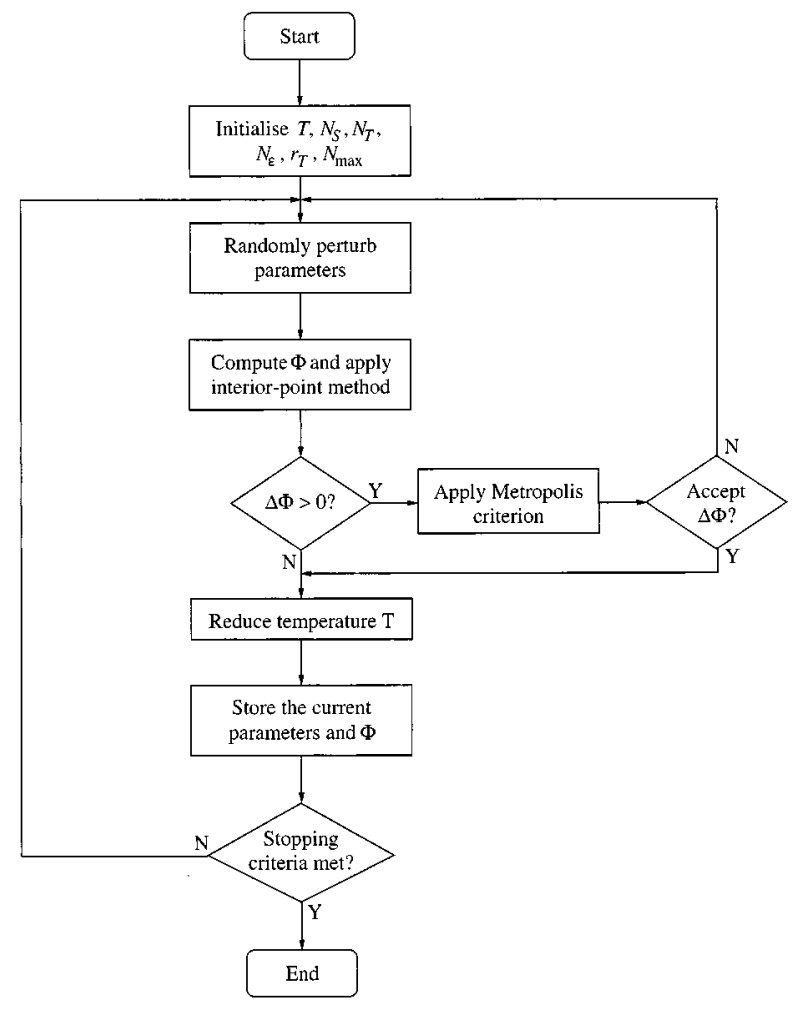

Fig. 2. Flowchart of the modified simulated annealing algorithm.

then perturbed randomly along each direction, in turn. If the perturbed parameter steps out of its boundaries, it is replaced by a value randomly generated within the boundaries. The cost function is then computed, and the probability of accepting the cost function is determined by (4). After $N_{s}(=20)$ steps through all parameters in $\boldsymbol{p}$, the step length vector is adjusted so that about $50 \%$ of the total number of moves are accepted. After $N_{T}$ $(=10 \times$ number of parameters $)$ step adjustments, the temperature $T$ is then reduced by a constant factor $r_{T}(=0.8)$. The algorithm terminates when 1) the differences between the recent $N_{\varepsilon}(=4)$ values of minima (including the current one) are less than a tolerance $\varepsilon\left(=10^{-5}\right)$ and 2$)$ the parameter changes are less than $0.1 \%$. These criteria help guarantee that the global minimum is reached. The algorithm also terminates when the maximum number of iterations $N_{\max }$ exceeds $10^{10}$. The above parameter settings were found to give satisfactory performance, although they might not be optimal.

\section{E. Computer Simulations}

To simulate ${ }^{99 \mathrm{~m}}$ Tc-teboroxime kinetics, a sum of two exponential decaying functions was used to generate an input function

$$
B(t)= \begin{cases}0, & t<0.3 \\ 2500 e^{-6(t-0.3)}+350 e^{-0.06(t-0.3)}, & t \geq 0.3\end{cases}
$$

where $t$ represents the time abscissa in minutes. Six tissue TACs of teboroxime were simulated with a two-compartment model. The parameter values were based on a baseline study obtained from a canine model [20], and a $70 \times 10$ s scanning protocol was assumed. Table I lists the values of the rate constants that were used for the simulations. Poisson noise typical of the observed 
TABLE I

Model Parameters Used to Simulate 99m Tc-Teboroxime Kinetics. NOMinal InITIAL VALUES, Which WeRE RANDOMIZED DURING SiMULATIONS, AND THE PHYSIOLOGICAL CONSTRAINTS IMPOSED ON THE PARAMETERS ARE ALSO SHOWN

\begin{tabular}{|c|c|c|c|c|c|c|c|c|c|}
\hline \multirow[b]{2}{*}{ Parameter } & \multicolumn{6}{|c|}{ Region } & \multirow{2}{*}{$\begin{array}{l}\text { Initial } \\
\text { value }\end{array}$} & \multicolumn{2}{|c|}{ Bound } \\
\hline & 1 & 2 & 3 & 4 & 5 & 6 & & lower & uppe \\
\hline$k_{21}\left(\min ^{-1}\right)$ & 0.590 & 1.109 & 0.482 & 0.760 & 0.856 & 0.652 & 0.5 & 0 & 10 \\
\hline$k_{12}\left(\min ^{-1}\right)$ & 0.229 & 0.295 & 0.236 & 0.255 & 0.272 & 0.236 & 0.5 & 0 & 10 \\
\hline$f_{v}$ (unitless) & 0.166 & 0.606 & 0.184 & 0.276 & 0.441 & 0.207 & 0.2 & 0 & 1 \\
\hline
\end{tabular}

TAC was added to the generated TACs, and 100 different noise realizations were simulated. To investigate the effect of initial value on the estimation of the IRFs and input function for both nonlinear least squares and simulated annealing, initial parameter estimates were randomized above a set of nominal initial values (Table I) as follows:

$$
p_{r}=p_{n} \times(1+0.5 X)
$$

where $X$ is a Gaussian distributed random variable with a mean of zero and a variance of one; $p_{n}$ is the nominal initial value of a parameter; and $p_{r}$ is the randomized parameter value. For a given parameter, if its randomized initial value was not within its boundaries, the randomization process was repeated until the boundary constraints were satisfied. The randomized initial values were then used by both nonlinear least squares and simulated annealing for the same realization.

For each region, the kinetic parameters were estimated by SIME in combination with nonlinear least squares or simulated annealing, in addition to the estimation of the input function scaled with two "blood" samples ( 8 and $11 \mathrm{~min}$ ) by minimizing (3) and were constrained to within their physiological ranges by the interior-point method [25]. The mean and standard deviation (SD) of the kinetic parameters were derived from the 100 noise realizations. The mean absolute difference (MAD) between an estimated parameter $\hat{p}^{i}$ and its true value $p^{i}$ in each of the $n$ tissue TACs was used as a measure of bias [26]

$$
\frac{1}{n} \sum_{i=1}^{n}\left|\hat{p}^{i}-p^{i}\right|
$$

and was computed over 100 realizations with different numbers of regions and initial estimates. All calculations were peformed on an UltraSPARC-2 workstation (296-MHz CPU, $256 \mathrm{MB}$ of memory).

\section{RESULTS AND DISCUSSION}

Fig. 3 shows a typical plot of the cost function value over the course of minimization using simulated annealing. A large variation of cost function value was observed initially due to energy changes caused by random perturbation on the parameters. There were some increases in the cost function during minimization because uphill moves were also accepted, according to the Metropolis criterion [16]. This is in contrast to iterative gradient-based techniques, where only downhill moves are accepted. However, as $K_{B} T$ decreases, positive changes in the cost function (i.e., $\Delta \Phi>0$ ) become less probable. After a large number of iterations, the minimum of the cost function

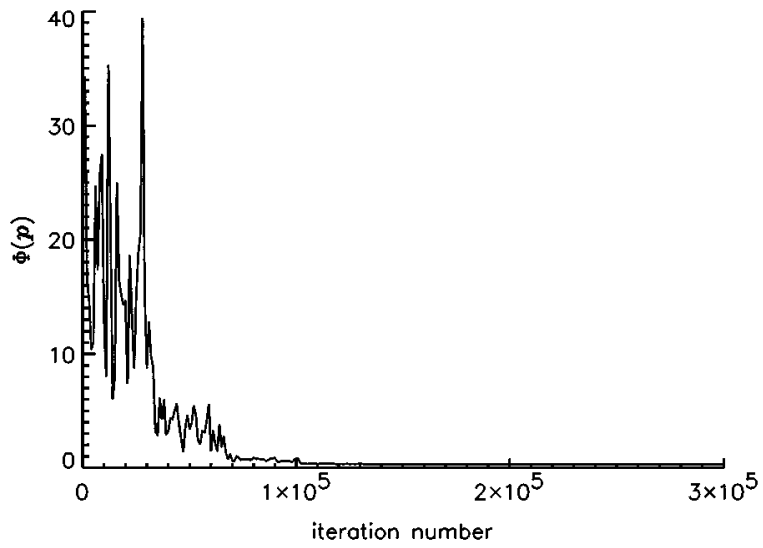

Fig. 3. Variation of cost function over the course of minimization.

TABLE II

PARAMETER ESTIMATES ObTAINEd USING NONLINEAR LEAST SQUARES (NLLS) AND SIMULATED ANNEALING (SA)

\begin{tabular}{llccc}
\hline & & & \multicolumn{2}{c}{ Parameter estimate } \\
\cline { 3 - 5 } Parameter & Region & True & NLLS & SA \\
\hline$k_{21}$ & 1 & 0.590 & $0.667 \pm 0.287$ & $0.577 \pm 0.056$ \\
$\left(\min ^{-1}\right)$ & 2 & 1.109 & $2.630 \pm 0.853$ & $1.095 \pm 0.800$ \\
& 3 & 0.482 & $0.499 \pm 0.151$ & $0.450 \pm 0.046$ \\
& 4 & 0.760 & $0.926 \pm 0.621$ & $0.756 \pm 0.093$ \\
& 5 & 0.856 & $1.337 \pm 0.703$ & $0.893 \pm 0.188$ \\
& 6 & 0.652 & $0.786 \pm 0.501$ & $0.656 \pm 0.073$ \\
\hline$k_{12}$ & 1 & 0.229 & $0.223 \pm 0.044$ & $0.218 \pm 0.017$ \\
$\left(\min ^{-1}\right)$ & 2 & 0.295 & $0.246 \pm 0.059$ & $0.289 \pm 0.032$ \\
& 3 & 0.236 & $0.219 \pm 0.032$ & $0.213 \pm 0.023$ \\
& 4 & 0.255 & $0.269 \pm 0.041$ & $0.265 \pm 0.020$ \\
& 5 & 0.272 & $0.261 \pm 0.039$ & $0.289 \pm 0.025$ \\
& 6 & 0.236 & $0.226 \pm 0.030$ & $0.234 \pm 0.022$ \\
\hline$f_{v}$ & 1 & 0.166 & $0.329 \pm 0.161$ & $0.196 \pm 0.056$ \\
& 2 & 0.606 & $0.879 \pm 0.299$ & $0.604 \pm 0.120$ \\
& 3 & 0.184 & $0.275 \pm 0.146$ & $0.173 \pm 0.050$ \\
& 4 & 0.276 & $0.414 \pm 0.210$ & $0.238 \pm 0.082$ \\
& 5 & 0.441 & $0.711 \pm 0.250$ & $0.455 \pm 0.107$ \\
& 6 & 0.207 & $0.425 \pm 0.194$ & $0.236 \pm 0.066$ \\
\hline Values are expressed as mean \pm SD. &
\end{tabular}

was located and the temperature was almost freezing. This can be considered analogous to the perfect crystal formation, where all molecules arrange themselves into a minimum energy state in which no further energy change takes place.

The mean value and standard deviation for the kinetic parameters in each of the regions obtained from SIME using nonlinear least squares and simulated annealing over 100 noise realizations are shown in Table II. The mean values of the washout parameter $k_{12}$ estimated by nonlinear least squares and simulated annealing were consistent. However, the precision for the estimates obtained by nonlinear least squares was lower than for those obtained with simulated annealing. There was a large variation in the mean value and standard deviation for the wash-in parameter $k_{21}$ and the blood volume $f_{v}$ when nonlinear least squares was used. The parameter precision was generally improved when simulated annealing was used instead.

Fig. 4 plots the MADs and their standard deviations over 100 noise realizations for $k_{21}, k_{12}$, and $f_{v}$ parameters as a function of the number of regions. Very large fluctuations in parameter estimates obtained with nonlinear least squares are clearly evident. On the other hand, the estimation of physiological parameters using simulated annealing is very insensitive to noise, as the bias was much less than that obtained with the nonlinear least 

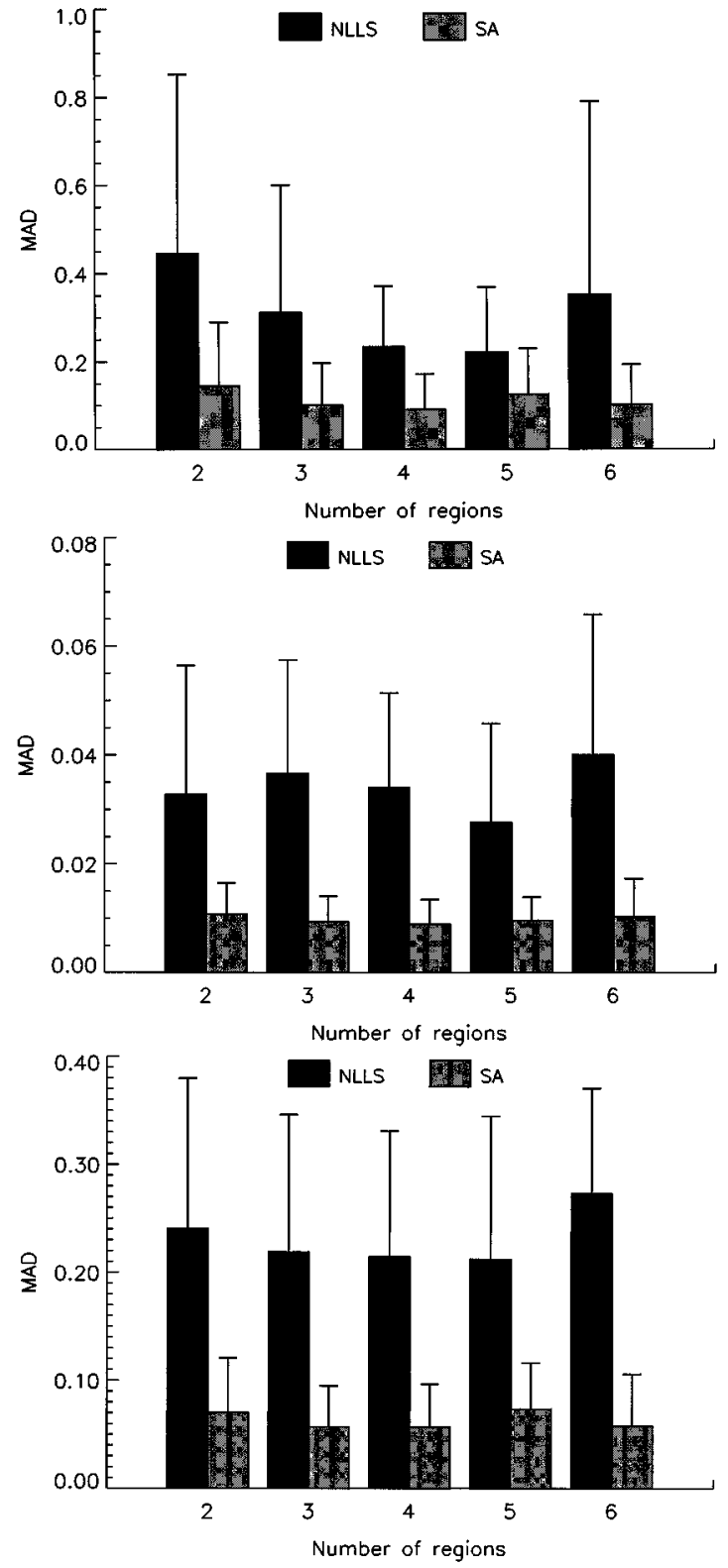

Fig. 4. MAD of (a) $k_{21}$, (b) $k_{12}$, and (c) $f_{v}$ as a function of number of regions. The standard deviation is represented by the error bar. (NLLS $=$ nonlinear least squares; $\mathrm{SA}=$ simulated annealing)

squares method starting with the same initial estimate and was almost the same with a different number of regions.

In general, incorporating more regions of distinct kinetics in SIME may provide more reliable and accurate recovery of the input function and estimation of kinetic parameters because the information of the input function increases. However, the identifiability of parameters may be degraded. The computational complexity is also higher with an increasing number of regions because the dimensionality of the cost function increases [12]. This then increases the difficulty in locating the best (global) optimum, particularly when nonlinear least squares is used. This is in contrast to simulated annealing, which provides estimates comparable to the true parameter values that were used to simulate the data. It is also interesting to see that the bias of parameter estimates did not improve noticeably with an increasing number of regions when simulated annealing was used (Fig. 4).
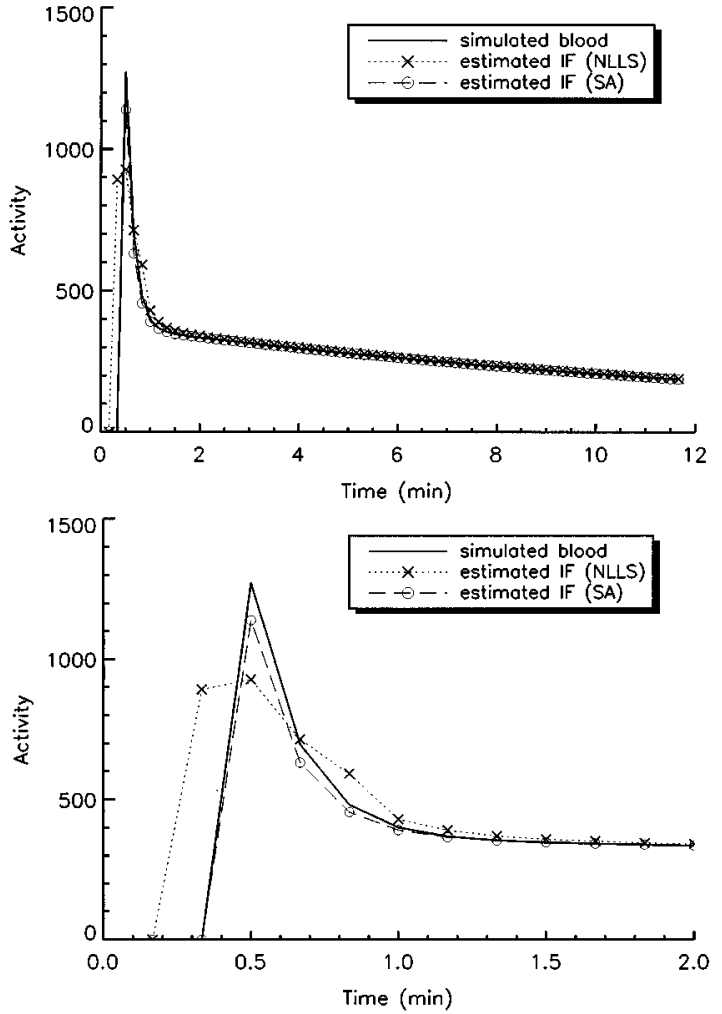

Fig. 5. (a) Simulated blood curve and estimated input functions using NLLS and SA. (b) Expanded plot of (a) for the first $2 \mathrm{~min}$.

It appears that two regions of distinct kinetics are sufficient to estimate the kinetic parameters and recover the input function if simulated annealing is used.

Fig. 5 shows the simulated blood curve and the estimated input functions over 100 noise realizations with four regions (Regions 1 to 4 ) obtained by simulated annealing and nonlinear least squares. The estimated input function obtained with simulated annealing closely resembled the simulated blood curve, while there was a biased estimation of the time delay and the peak of the input function when nonlinear least squares was used. This was due to the nonlinear least squares algorithm's being trapped in local minima because it allows only downhill moves, which follow the gradient of descent locally. Therefore, other optimal points with lower cost function values than the current point may not be reachable. On the contrary, simulated annealing occasionally accepts uphill moves, thus making it possible to escape local minima. In light of this, other optimization methods, such as genetic algorithms [27] and tabu search [28], that are able to move "uphill" similar to simulated annealing may also be worth investigating.

Inclusion of blood volume $f_{v}$ in the kinetic model has been proposed as a more accurate means to model the (in vivo) situation [29]. However, we found that the identifiability of the wash-in parameter $k_{21}$ deteriorated when $f_{v}$ was included because these parameters were highly correlated and were not uniquely identifiable even though the model fitting was improved. Faster temporal sampling might help in reducing the correlation between the parameters. However, the speed of mechanical rotation of the detectors limits the maximum sampling rate to $>5 \mathrm{~s} / \mathrm{sample}$ in modern SPECT systems. 
Even if the image statistics were adequate, the improvement in identifying $k_{21}$ and $f_{v}$ might not be significant. Simulated annealing worked reasonably well in the estimation of $k_{21}$ when compared to the nonlinear least squares in terms of parameter bias and reliability because it updates the parameter estimates sequentially in place rather than considering all the parameters simultaneously. The convergence rate of simulated annealing was also found to be much faster when $f_{v}$ was not included in the compartment model. This is not surprising because $k_{21}$ and $k_{12}$ are uniquely identifiable, although they may be biased. In spite of the larger standard deviation (lower reliability) in the parameter estimate, the estimation of $k_{12}$ did not vary appreciably even if $f_{v}$ was included, regardless of which minimization method was used.

Recent work by Di Bella et al. [10] has some similarities to our previous work [8], [12]. Their approach applied the crosscorrelation method [30] to solve an overdetermined system of output equations in the frequency domain. A scaling factor is thus required for unique quantification of kinetic parameters. Their approach was tested on uniformly sampled data, though it is not an essential requirement, as the nonuniformly sampled data can be interpolated into uniformly sampled grids. However, the errors introduced by the interpolation and their effects on the kinetic parameter estimation are not known. Our proposed method, on the other hand, has the advantages that 1) nonuniformly sampled data can be accommodated without resampling the data and 2) the least squares minimization is performed in the time domain. This avoids using fast Fourier transform, which may produce erroneous results caused by aliasing when the data are undersampled. Nonetheless, it is important that a carefully designed sampling protocol be used to optimize the accuracy and reliability of the parameter estimates.

The assumption of an identical input function that is common to the tissue TACs in all regions may need to be considered before applying SIME and the method of Di Bella et al. [10]. Since the actual input functions seen from distant regions may not be identical due to differences in tracer delivery in blood, both of the methods may produce an incorrect estimation of the underlying input function. Identifiability of the input function may be improved by including regions drawn, either manually or automatically (e.g., by factor analysis [4], [11] or cluster analysis [31], [32]), over vascular structures in the model-fitting procedure. Nonetheless, corrections for time discrepancies between tissue curves and input curves extracted from vascular structures, spillover of activity from surrounding tissues into blood, and motion artifacts, should be implemented cautiously in order to estimate kinetic parameters accurately [33].

Noise in the measured input function leads to statistical uncertainties in the model-predicted output and the kinetic parameters. Methods have been developed to analyze kinetic data with a noisy input function [34]-[36]. In particular, Huesman [37] suggested that the result obtained by estimating the input function from measured input data is equivalent to that of not estimating the input function but using the measured input function directly for compartment model fitting. In other words, the aim of such methods can be loosely considered as a minimization of a sum of two objective functions: one that minimizes the weighted differences between the tissue measurement and the model prediction, as in conventional compartment model fitting, and another that is used to discourage the differences between the measured input function and its expected values. However, the main advance of this study is that the expected input function is recovered from the tissue measurements rather than from the noisy input function, whose time course is only known at one or two time instants. Thus, more parameters must be estimated. The second objective function can only discourage the differences between the measured and the expected input function at the time where blood measurements are available, while the expected input function is also used in the first objective function for minimization.

Choosing an appropriate set of initial estimates is very important for kinetic modeling when an iterative gradient-based technique is used. Any effort spent in obtaining good initial estimates of the parameters is well rewarded by rapid convergence of the minimization algorithm and reduced chance of straying into an incorrect, local minimum. Incorporating ( $a$ priori) information about the input function and the kinetic parameter estimates from theory or previous experimental results, and shrinking the parameter space by constraining the parameter estimates to within certain limits, are usually helpful. However, clinical data are always compounded by noise, which perturb the smoothness of the cost function surface and create a number of minima. Even though the constraints may be satisfied, this does not guarantee the global optimality of the parameter estimates.

Although this study considered only the case of teboroxime, which is a flow-dependent tracer with fast kinetics, the method may work equally well in other radiopharmaceuticals with rapid kinetics predominantly dependent on flow. In the case of FDG or receptor studies, the tissue data may have less information about the shape of the input function. The ability to recover the input function and kinetic parameter estimation for those studies may not be as good as that of flow-dependent radiopharmaceuticals. Application of simulated annealing with SIME to those studies deserves further investigation.

One of the major problems associated with simulated annealing is the large number of parameters to be adjusted in order to give optimal performance. Of particular importance is the cooling schedule that governs how the temperature is decreased. It has a major impact on the speed of convergence of the algorithm and the optimality of the parameter estimates. If the temperature is quickly reduced, the algorithm may be trapped at a suboptimal point. On the other hand, long computation times are required for the algorithm to converge to a minimum if the temperature decreases too slowly. There are no general guidelines for selecting a cooling schedule, and this remains an active area of research.

Widespread application of simulated annealing in parameter estimation has been limited because of the intensive computational burden required. Typically, the nonlinear least squares method required about $30 \mathrm{~s}$ to minimize (3), but approximately 15 min was required for simulated annealing. Nonetheless, in the case of very ill-conditioned cost functions with many local minima (e.g., problems with many parameters), the expense in computation may be rewarded by obtaining better results than the nonlinear least squares method, which may need to be restarted at different points many times. In addition, simulated annealing can still be applicable to problems where the cost 
function cannot be approximated by a quadratic function near the minimum or is nondifferentiable or discontinuous in its domain. Searching for minima with gradient-based methods in this case may not be feasible. Further, continuing advances in computer technology, together with the use of parallel processors, are diminishing computational burden as a limiting factor.

\section{CONCLUSION}

Our results demonstrate that it is feasible to apply simulated annealing to estimate the physiological parameters and the input function simultaneously in a flow model. It is more insensitive to noise than the nonlinear least squares method. The physiological parameters obtained with simulated annealing are more accurate, and the estimated input function more closely resembled the simulated curve. We conclude that simulated annealing reduces bias in the estimation of physiological parameters and determination of the input function, and further research on its application to radiopharmaceuticals that are not as critically dependent on the shape of the input function is warranted.

\section{REFERENCES}

[1] S. C. Huang and M. E. Phelps, "Principles of tracer kinetic modeling in positron emission tomography and autoradiography," in Positron Emission Tomography and Autoradiography: Principles and Applications for the Brain and Heart, M. E. Phelps, J. C. Mazziotta, and H. R. Schelbert, Eds. New York: Raven, 1986, pp. 287-346.

[2] I. N. Weinberg, S. C. Huang, E. J. Hoffman, L. Araujo, C. Nienaber M. Grover-McKay, M. Dahlbom, and H. R. Schelbert, "Validation of PET-acquired input functions for cardiac studies," J. Nucl. Med., vol. 29, pp. 241-247, 1988

[3] A. D. Nelson, F. Miraldi, R. F. Muzic Jr., G. P. Leisure, and W. E. Semple, "Noninvasive arterial monitor for quantitative oxygen-15-water blood flow studies," J. Nucl. Med., vol. 34, pp. 1000-1006, 1993.

[4] H. M. Wu, C. K. Hoh, D. B. Buxton, H. R. Schelbert, Y. Choi, R. A Hawkins, M. E. Phelps, and S. C. Huang, "Factor analysis for extraction of blood time-activity curves in dynamic FDG-PET studies," $\mathrm{J}$. Nucl. Med., vol. 36, pp. 1714-1722, 1995.

[5] Y. Onishi, Y. Yonekura, S. Nishizawa, F. Tanaka, H. Okazawa, K. Ishiza, T. Fujita, J. Konishi, and T. Mukai, "Noninvasive quantification of iodine-123-iomazenil SPECT," J. Nucl. Med., vol. 37, no. 2, pp. 374-378, 1996.

[6] H. Watabe, M. Itoh, V. Cunningham, A. A. Lammertsma, P. M. Bloomfield, M. Mejia, T. Fujiwara, A. K. P. Jones, T. Jones, and T. Nakamura, "Non-invasive quantification of $r \mathrm{CBF}$ using positron emission tomography," J. Cereb. Blood Flow Metab., vol. 16, pp. 311-319, 1996.

[7] S. Eberl, A. R. Anayat, R. R. Fulton, P. K. Hooper, and M. J. Fulham, "Evaluation of two population-based input functions for quantitative neurological FDG PET studies," Eur. J. Nucl. Med., vol. 24, no. 3, pp. 299-304, 1997.

[8] D. Feng, K. P. Wong, C. M. Wu, and W. C. Siu, "A technique for extracting physiological parameters and the required input function simultaneously from PET image measurements: Theory and simulation study," IEEE Trans. Inform. Technol. Biomed., vol. 1, pp. 243-254, Dec. 1997.

[9] K. Chen, D. Bandy, E. Reiman, S. C. Huang, M. Lawson, D. Feng, L. S. Yun, and A. Palant, "Noninvasive quantification of the cerebral metabolic rate for glucose using positron emission tomography, ${ }^{18}$ F-fluoro-2-deoxyglucose, the Patlak method, and an image-derived input function," J. Cereb. Blood Flow Metab., vol. 18, pp. 716-723, 1998.

[10] E. V. R. Di Bella, R. Clackdoyle, and G. T. Gullberg, "Blind estimation of compartmental model parameters," Phys. Med. Biol., vol. 44, pp. 765-780, 1999.

[11] A. Sitek, E. V. R. Di Bella, and G. T. Gullberg, "Factor analysis with a priori knowledge-Application in dynamic cardiac SPECT," Phys. Med. Biol., vol. 45, pp. 2619-2638, 2000.
[12] K. P. Wong, D. Feng, S. R. Meikle, and M. J. Fulham, "Simultaneous estimation of physiological parameters and the input function-In vivo PET data," IEEE Trans. Inform. Technol. Biomed., vol. 5, pp. 67-76, Mar. 2001.

[13] M. J. D. Powell, "An efficient method for finding the minimum of a function of several variables without calculating derivatives," Comput. J., vol. 7, pp. 155-162, 1964.

[14] R. Hooke and T. A. Jeeves, "Direct search solution of numerical and statistical problems," J. Assoc. Comput. Mach., vol. 8, pp. 212-229, 1961.

[15] J. A. Nelder and R. Mead, "A simplex method for function minimization," Comput. J., vol. 7, pp. 308-313, 1965.

[16] S. Kirkpatrick, C. D. Gelatt Jr., and M. P. Vecchi, "Optimization by simulated annealing," Science, vol. 220, pp. 671-680, 1983

[17] W. E. Smith, R. G. Paxman, and H. H. Barrett, "Application of simulated annealing to coded-aperture design and tomographic reconstruction," IEEE Trans. Nucl. Sci., vol. NS-32, pp. 758-761, 1985.

[18] S. Webb, "SPECT reconstruction by simulated annealing," Phys. Med. Biol., vol. 34, pp. 259-281, 1989.

[19] K. A. Girodias, H. H. Barrett, and R. L. Shoemaker, "Parallel simulated annealing for emission tomography," Phys. Med. Biol., vol. 36, pp. 921-938, 1991.

[20] A. M. Smith, G. T. Gullberg, P. E. Christian, and F. L. Datz, "Kinetic modeling of teboroxime using dynamic SPECT imaging of a canine model," J. Nucl. Med., vol. 35, pp. 484-495, 1994.

[21] R. E. Stewart, M. Schwaiger, G. D. Hutchins, P. C. Chiao, K. P. Gallagher, N. Nguyen, N. A. Petry, and W. L. Rogers, "Myocardial clearance kinetics of technetium-99m-SQ30217: A marker of regional myocardial blood flow," J. Nucl. Med., vol. 31, pp. 1183-1190, 1990.

[22] D. W. Marquardt, "An algorithm for least-squares estimation of nonlinear parameters," J. Soc. Ind. Appl. Math., vol. 11, pp. 431-441, 1963.

[23] N. Metropolis, A. Rosenbluth, M. Rosenbluth, A. Teller, and E. Teller, "Equation of state calculations by fast computing machines," J. Chem. Phys., vol. 21, pp. 1087-1092, 1953.

[24] A. Corana, M. Marchesi, C. Martini, and S. Ridella, "Minimizing multimodal functions of continuous variables with the "simulated annealing" algorithm," ACM Trans. Math. Software, vol. 13, pp. 262-280, 1987.

[25] A. V. Fiacco and G. P. McCormick, Nonlinear Programming: Sequential Unconstrained Minimization Techniques. New York: Wiley, 1968.

[26] A. Welch, A. M. Smith, and G. T. Gullberg, "An investigation of the effect of finite system resolution and photon noise on the bias and precision of dynamic cardiac SPECT parameters," Med. Phys., vol. 22, pp. 1829-1836, 1995.

[27] D. E. Goldberg, Genetic Algorithms in Search, Optimization, and Machine Learning. Reading, MA: Addison-Wesley, 1989.

[28] F. Glover and M. Laguna, Tabu Search. Norwell, MA: Kluwer, 1997.

[29] A. A. Lammertsma, D. J. Brooks, R. S. J. Frackowiak, R. P. Beaney, S Herold, J. D. Heather, A. J. Palmer, and T. Jones, "Measurement of glucose utilization with $\left[{ }^{18} \mathrm{~F}\right] 2$-fluoro-2-deoxy-D-glucose: A comparison of different analytical methods," J. Cereb. Blood Flow Metab., vol. 7, pp. $161-172,1987$.

[30] G. Xu, H. Liu, L. Tong, and T. Kailath, "A least-squares approach to blind channel identification," IEEE Trans. Signal Processing, vol. 43, pp. 2982-2993, Dec. 1995.

[31] F. O'Sullivan, "Imaging radiotracer model parameters in PET: A mixture analysis approach," IEEE Trans. Med. Imaging, vol. 12, pp. 399-412, Sept. 1993.

[32] K. P. Wong, D. Feng, S. R. Meikle, and M. J. Fulham, "Segmentation of dynamic PET images using cluster analysis," IEEE Trans. Nucl. Sci., vol. 49, pp. 206-207, Feb. 2002.

[33] P. Herrero, J. J. Hartman, M. J. Senneff, and S. R. Bergmann, "Effects of time discrepancies between input and myocardial time-activity curves on estimates of regional myocardial perfusion with PET," J. Nucl. Med., vol. 35, pp. 558-566, 1994.

[34] R. H. Huesman and B. M. Mazoyer, "Kinetic data analysis with a noisy input function," Phys. Med. Biol., vol. 32, pp. 1569-1579, 1987.

[35] K. Chen, S. C. Huang, and D. C. Yu, "The effects of measurement errors in plasma radioactivity curve on parameter estimation in positron emission tomography," Phys. Med. Biol., vol. 36, no. 9, pp. 1183-1200, 1991.

[36] P. C. Chiao, W. L. Rogers, N. H. Clinthorne, J. A. Fessler, and A. O. Hero, "Model-based estimation for dynamic cardiac studies using ECT," IEEE Trans. Med. Imaging, vol. 13, pp. 217-226, June 1994.

[37] R. H. Huesman, "Equivalent methods to analyze dynamic experiments in which the input function is noisy," Phys. Med. Biol., vol. 42, pp. 147-153, 1997. 